Louisiana State University

LSU Digital Commons

4-1-2016

\title{
Enhanced spectral sensitivity of a chip-scale photonic-crystal slow-light interferometer
}

\author{
Omar S. Magaña-Loaiza \\ University of Rochester Institute of Optics \\ Boshen Gao \\ University of Rochester Institute of Optics \\ Sebastian A. Schulz \\ University of Ottawa \\ Kashif M. Awan \\ University of Ottawa \\ Jeremy Upham \\ University of Ottawa
}

See next page for additional authors

Follow this and additional works at: https://digitalcommons.Isu.edu/physics_astronomy_pubs

\section{Recommended Citation}

Magaña-Loaiza, O., Gao, B., Schulz, S., Awan, K., Upham, J., Dolgaleva, K., \& Boyd, R. (2016). Enhanced spectral sensitivity of a chip-scale photonic-crystal slow-light interferometer. Optics Letters, 41 (7), 1431-1434. https://doi.org/10.1364/OL.41.001431

This Article is brought to you for free and open access by the Department of Physics \& Astronomy at LSU Digital Commons. It has been accepted for inclusion in Faculty Publications by an authorized administrator of LSU Digital Commons. For more information, please contact ir@lsu.edu. 


\section{Authors}

Omar S. Magaña-Loaiza, Boshen Gao, Sebastian A. Schulz, Kashif M. Awan, Jeremy Upham, Ksenia Dolgaleva, and Robert W. Boyd 


\title{
Enhanced spectral sensitivity of a chip-scale photonic-crystal slow-light interferometer
}

\author{
Omar S. Magaña-Loaiza, ${ }^{1, *}$ Boshen Gao, ${ }^{1}$ Sebastian A. Schulz, ${ }^{2}$ Kashif M. Awan, ${ }^{3}$ Jeremy Upham, ${ }^{2}$ \\ Ksenia Dolgaleva, ${ }^{2,3}$ and Robert W. Boyd ${ }^{1,2,3}$ \\ ${ }^{1}$ The Institute of Optics, University of Rochester, Rochester, New York 14627, USA \\ ${ }^{2}$ Department of Physics and Max Planck Centre for Extreme and Quantum Photonics, University of Ottawa, 25 Templeton, Ottawa, ON, K1N 6N5, \\ Canada \\ ${ }^{3}$ School of Electrical Engineering and Computer Science, University of Ottawa, 25 Templeton, Ottawa, ON, K1N 6N5, Canada \\ ${ }^{*}$ Corresponding author: omar.maganaloaiza@rochester.edu
}

Received 29 January 2016; revised 19 February 2016; accepted 23 February 2016; posted 26 February 2016 (Doc. ID 256749); published 21 March 2016

We experimentally demonstrate that the spectral sensitivity of a Mach-Zehnder (MZ) interferometer can be enhanced through structural slow light. We observe a 20 -fold resolution enhancement by placing a dispersion-engineered, slow-light, photonic-crystal waveguide in one arm of a fiber-based MZ interferometer. The spectral sensitivity of the interferometer increases roughly linearly with the group index, and we have quantified the resolution in terms of the spectral density of interference fringes. These results show promise for the use of slow-light methods for developing novel tools for optical metrology and, specifically, for compact high-resolution spectrometers. (C) 2016 Optical Society of America

OCIS codes: (120.0120) Instrumentation, measurement, and metrology; (120.3180) Interferometry; (050.5298) Photonic crystals.

http://dx.doi.org/10.1364/OL.41.001431

Slow light has fascinated the physics community for over two decades [1,2]. The slowdown of light has been observed in a diverse range of media, including atomic vapors, optical fibers, and photonic crystals (PhCs) [3]. The ability to manipulate the speed of light has led to a wide variety of technological applications, for example, optical buffers, optical memories, optical modulators, laser radars, and enhanced spectrometers $[1,2,4-9]$.

Optical interferometry has benefited greatly from the use of highly dispersive materials [10-17]. For instance, it has been predicted that a Mach-Zehnder (MZ) interferometer with a slow-light medium in one of the arms will greatly enhance spectral sensitivity with respect to its conventional (non-slow light) version $[10,14,15]$. This result holds promise for compact and efficient spectrometers $[1,9]$. Nevertheless, up to this date, the improvement of the spectral sensitivity of interferometers has been demonstrated only in bulk optical setups, such as shearing and free-space interferometers, with highly dispersive crystals and atomic vapors [14-16].
While not achieving the same extreme slowdown values as material slow light, slow light in nanophotonic devices, particularly in photonic-crystal (PhC) waveguides, has many key advantages. Nanophotonic slow-light structures are extremely compact, with low footprint and high mechanical stability, compared to material slow-light systems [3]. Furthermore, the availability of high-precision fabrication techniques and the use of common materials, e.g., silicon, makes nanophotonic slow light particularly interesting for applications [3-5,7]. Here we demonstrate that structural slow light can dramatically improve the spectral sensitivity of an interferometer. Thus, we combine the enhancement to the spectral sensitivity with the advantages of nanophotonics. Specifically, we place a silicon $\mathrm{PhC}$ slow-light waveguide within a compact fiber-based $\mathrm{MZ}$ interferometer; see Fig. 1. Our results show an enhancement of a factor of 20 in the spectral sensitivity of the interferometer, without increasing its physical size. This sensitivity is on par with that of previous implementations of material slow-light interferometers [14], using a much more compact slow-light structure (the total $\mathrm{PhC}$ footprint is on the order of $0.015 \mathrm{~mm}^{2}$ ) [14-16]. To demonstrate the advantage of adding a slow-light medium into a spectral interferometer, we discuss the phase difference $\Delta \phi$ between two light beams passing through the two arms of a $\mathrm{MZ}$ interferometer. We assume that the phase difference is solely caused by propagation through a length $L$ of slow-light material. The phase difference is defined as

$$
\Delta \phi=\frac{\omega}{c} n(\omega) L
$$

where $\omega$ is the frequency of the propagating light, $c$ is the speed of light in vacuum, and $n(\omega)$ is the refractive index of the medium. In practice, $\Delta \phi$ is determined by performing intensity measurements of the interference pattern produced at one of the output ports of the interferometer:

$$
I=\frac{I_{0}}{2}(1+\cos \Delta \phi)
$$

where $I_{0}$ is the input intensity. The spectral resolution of the interferometer is determined by the minimum resolvable change 
a)

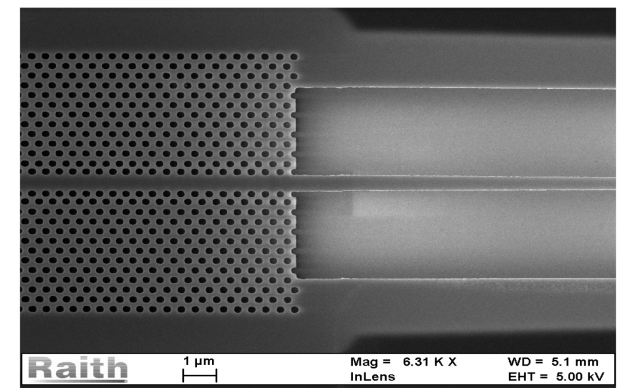

b)

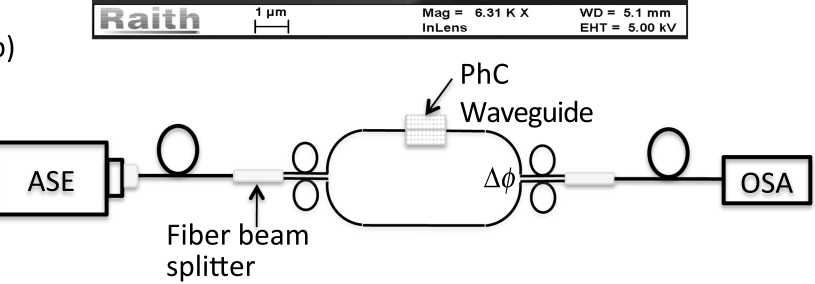

Fig. 1. Slow-light waveguide and measurement setup. (a) Scanning electron microscope (SEM) photograph of the fabricated PhC waveguide and its access channel. (b) Fiber-based Mach-Zehnder interferometer containing a $\mathrm{PhC}$ slow-light waveguide in one of the arms. In addition, we utilize a broadband amplified spontaneous emission (ASE) source and an optical spectrum analyzer (OSA). The difference in optical path length achieved through the imbalance between the two arms produced by the slow-light region is indicated by $\Delta \phi$.

in intensity due to a shift in frequency [18]. This change is quantified by the first derivative of the intensity with respect to the frequency:

$$
\frac{d I}{d \omega}=\frac{I_{0}}{2} \frac{d \Delta \phi}{d \omega} \sin \Delta \phi=\frac{I_{0}}{2} \frac{L}{c}\left(n+\omega \frac{d n}{d \omega}\right) \sin \Delta \phi .
$$

We note that $n+\omega d n / d \omega$ is the definition of the group index, $n_{g}$. Thus, Eq. (3) can be rewritten as

$$
\frac{d I}{d \omega}=\frac{I_{0}}{2} \frac{L n_{g}}{c} \sin \Delta \phi .
$$

We see that a larger group index leads to denser fringes which, in turn, leads to the enhancement of the spectral sensitivity of the interferometer [18]. This enhancement depends on the product $n_{g} L$. Thus, using a slow-light medium with a high $n_{g}$ reduces the physical size of the interferometer needed to achieve a given spectral sensitivity. The group index, $n_{g}$, can be significantly higher than the refractive index, $n$, of a material and, therefore, slow-light materials can significantly enhance the spectral sensitivity of interferometers $[1,2,9]$. In our experiment, we measure the interference pattern formed at the output of the MZ interferometer as a function of wavelength $\lambda$. This measurement allows us to quantify the enhancement in resolution and sensitivity through an experimental parameter, the density of fringes per unit wavelength interval, $N_{\lambda}$. An approximate expression for the local density of fringes over a small frequency range $\Delta \omega$ can be derived by describing the refractive index of the dispersive material as $n \approx n_{0}+\left(n_{g}(\lambda)-n_{0}\right) \Delta \omega$, where it is assumed that $n_{g}$ remains approximately constant over the narrow frequency range being considered. Since one fringe represents a net change of phase difference of $2 \pi$, the period of one fringe in frequency domain, $\Delta \omega_{0}$, is determined by solving $L / c\left(n_{0} \Delta \omega_{0}+\left(n_{g}-n_{0}\right) \Delta \omega_{0}^{2}\right)=2 \pi$. This, together with the relation between wavenumber and frequency, allows us to define the local fringe density as

$$
N_{\lambda}=\frac{\sqrt{n_{0}^{2} L^{2}+8\left\{n_{g}(\lambda)-n_{0}\right\} \pi c L}+n_{0} L}{2 \lambda^{2}} .
$$

For a non-dispersive medium $\left(n_{g}=n_{0}\right)$, this equation recovers the result for a traditional free-space interferometer, $N_{\lambda}=n_{0} L / \lambda^{2}$. For a slow-light medium, where $n_{g}>n_{0}$, the fringe density is increased. Thus, from Eq. (5), we expect a reduction in the fringe density with increasing wavelength and an increase in the fringe density with increasing group index. We recall that the group index itself is strongly wavelength dependent and increases monotonically with wavelength in the spectral region considered here.

We engineered the dispersive properties of our W1-based $\mathrm{PhC}$ waveguides through a shift of the first and second rows of holes perpendicular to the waveguide direction [19]. We also include coupling interfaces to maximize the transmission [20]. The waveguide has a lattice constant of $416 \mathrm{~nm}$ and a hole radius of $124 \mathrm{~nm}$. The silicon slab is $210 \mathrm{~nm}$ thick, and the first and second rows are shifted by $46 \mathrm{~nm}$ outward and $16 \mathrm{~nm}$ inward, respectively. The waveguide was fabricated according to the procedure described in [21]. A scanning electron microscope (SEM) image of the device is shown in Fig. 1(a).

The slow-light characteristics of the device were determined through Fourier transform spectral interferometry [22]. Due to the nature of our slow-light material and its mechanical stability, we chose a fiber-based $\mathrm{MZ}$ interferometer with the slow-light waveguide in one of the arms. This setup is used as a proofof-principle experiment to demonstrate the enhancement of the sensitivity due to structural slow light; see Fig. 1(b). Light is coupled into and out of the waveguide using grating couplers that were etched at the same time as the $\mathrm{PhC}$ waveguide; see Fig. 1(a). The optical output of the slow-light MZ interferometer is measured as a function of wavelength through the use of an optical spectrum analyzer (OSA). We use a broadband, amplified spontaneous emission (ASE) source spanning the wavelength range from 1525 to $1575 \mathrm{~nm}$. The obtained interference pattern is shown in Figs. 2(a)-2(d). The modulation of the fringe height is due to a resonant effect caused by reflections from the grating couplers of the PhC sample and is not intrinsic to a slow-light interferometer. The increase in fringe density with wavelength is a consequence of the slow-light effect in the $\mathrm{PhC}$ waveguide. The experimental measurement, Fig. 2(e), shows the increase in group index as a function of wavelength. As predicted by Eqs. 4 and 5, when the dispersive properties of the material become significant, they lead to an enhancement of the resolution and sensitivity of the device.

In Fig. 3(a), we show the experimental characterization of the enhancement in sensitivity of our interferometer. We plot the fringe density of the slow-light MZ interferometer as a function of wavelength. The increased density at longer wavelength leads to an increase in the spectral resolution of the device. The red curve in Fig. 3(a) shows the fringe density predicted by our numerical simulation using a plane wave expansion approach (MPB [23]). Further, the expected fringe density of a traditional $\left(n_{g} \approx n\right) \mathrm{MZ}$ interferometer is plotted in green. Clearly, there is a dramatic improvement in the fringe density and, therefore, in the sensitivity of the interferometer, when a slow-light structure is incorporated within it.

For this particular $\mathrm{PhC}$ waveguide, our proof-of-principle experiment shows an increase of 20 times in the sensitivity. This number is taken from Fig. 3(a) by calculating the ratio 

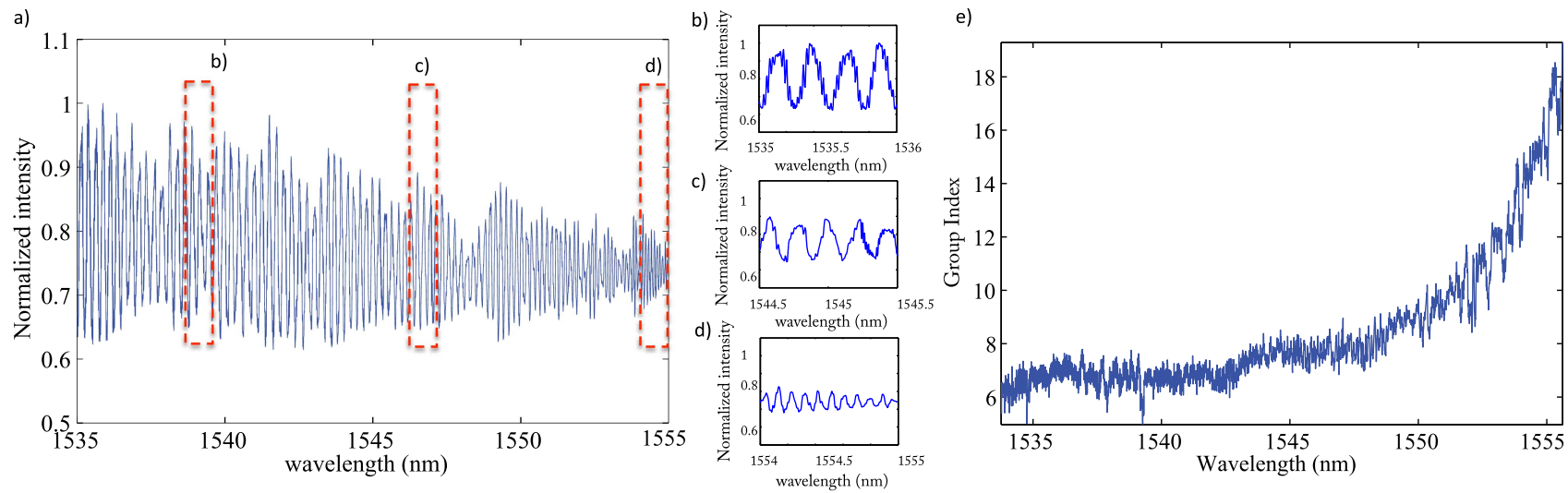

Fig. 2. (a) Intensity of one of the outputs of the MZ interferometer plotted as a function of wavelength. Note that the fringe density increases with increasing wavelength. Insets (b)-(d) show three different $1 \mathrm{~nm}$ spans with significantly different fringe spacings. (e) Wavelength dependence of the group index.

between the fringe density for the interferometer with (curve in red) and without (curve in green) the dispersive medium at a wavelength of $1556 \mathrm{~nm}$. Further evidence of the role of the group index in determining the sensitivity of the interferometer
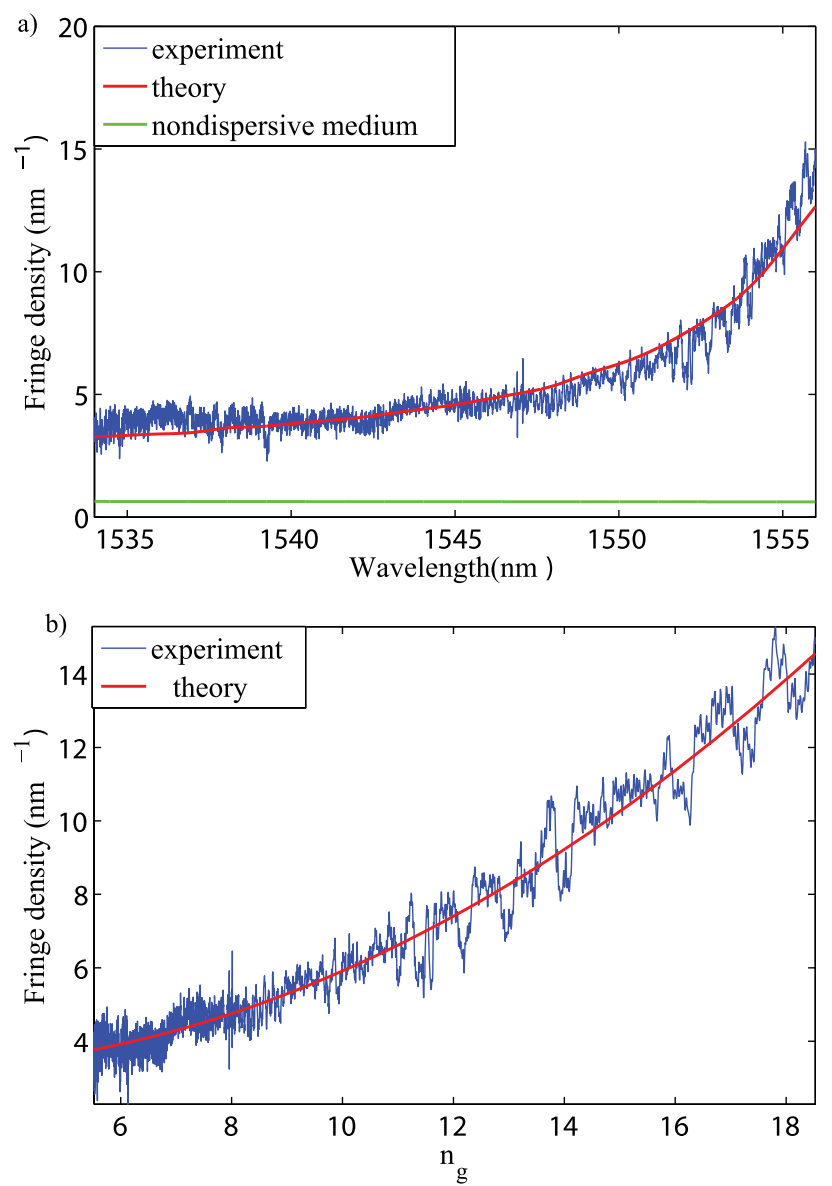

Fig. 3. (a) Experimental measurements and theoretical predictions of the density of interference fringes plotted as a function of wavelength for the slow-light MZ interferometer of Fig. 1(a). (b) Dependence of fringe density on group index $n_{g}$ for the same waveguide. We see that the fringe density and, consequently, the spectral sensitivity, increases with the group index of the slow-light waveguide. is shown in Fig. 3(b). In this case, the increased resolution of the device, quantified by its fringe density, exhibits a direct relationship with the group index, as described by Eqs. 3-5. This group index dependence allows one to keep the physical size of interferometers small, creating the potential for compact spectrometers. The spectral sensitivity could be optimized for different applications by engineering the $\mathrm{PhC}$ waveguide properties. For instance, further increasing the resolution or creating spectral ranges with a constant enhancement.

In summary, we have demonstrated an enhancement of the spectral resolution, accompanied by enhanced sensitivity, of a MZ interferometer by incorporating a structural slow-light component, in our case, a $\mathrm{PhC}$ waveguide. This enhancement, which reached a factor of 20 in our experiment, can be further improved through careful waveguide design. We demonstrated the increased sensitivity through a significant increase in the density of interference fringes. Enhanced-sensitivity interferometers have been an important subject of interest in the photonics community for the last 10 years $[1,2,4,5,7,9,10,14-17]$. Therefore, we believe that our experimental results are an important starting point for further studies, including on-chip implementations of our device. A monolithic device of this sort would reduce losses and imperfections in our proof-of-principle design. These results provide a path toward a new generation of compact sensitive devices and sensors utilizing light.

Funding. National Aeronautics and Space Administration (NASA) (NNX15CM47P); Defense Threat Reduction Agency (DTRA), Joint Science and Technology Office for Chemical and Biological Defense (HDTRA1-10-1-0025); Canada Excellence Research Chairs Program.

Acknowledgment. The authors would like to thank David D. Smith and Jerry Kuper for many fruitful discussions.

\section{REFERENCES}

1. R. W. Boyd, J. Mod. Opt. 56, 1908 (2009).

2. R. W. Boyd and D. J. Gauthier, Science 326, 1074 (2009).

3. R. W. Boyd, J. Opt. Soc. Am. B 28, A38 (2011).

4. T. Baba, Nat. Photonics 2, 465 (2008).

5. T. F. Krauss, J. Phys. D 40, 2666 (2007). 
6. H. C. Nguyen, S. Hashimoto, M. Shinkawa, and T. Baba, Opt. Express 20, 22465 (2012).

7. T. F. Krauss, Nature Photon 2, 448 (2008).

8. A. Schweinsberg, J. E. Vornehm, and R. W. Boyd, Opt. Express 19, 15760 (2011).

9. Z. Zhi and R. W. Boyd, J. Opt. Soc. Am. B 25, 136 (2008).

10. M. Soljacic, S. G. Johnson, S. Fan, M. Ibanescu, E. Ippen, and J. D. Joannopoulos, J. Opt. Soc. Am. B 19, 2052 (2002).

11. Y. A. Vlasov, M. O'Boyle, H. F. Hamann, and S. J. McNab, Nature 438, 65 (2005).

12. C. Reimer, N. Nedelijkovic, D. J. M. Stothard, M. O. S. Esnault, C. Reardon, L. O'Faolain, M. Dunn, G. Z. Mashanovich, and T. F. Krauss, Opt. Express 20, 29361 (2012).

13. Y. Zhang, H. Tian, X. Zhang, N. Wang, J. Zhang, H. Wu, and P. Yuan, Opt. Lett. 35, 691 (2010).

14. Z. Zhi, R. W. Boyd, D. J. Gauthier, and C. C. Dudley, Opt. Lett. 32 2251 (2007).
15. Z. Shi, R. W. Boyd, R. M. Camacho, P. K. Vudyasetu, and J. C. Howell, Phys. Rev. Lett. 99, 240801 (2007).

16. J. Wang, Y. Zhang, Z. Xuenan, H. Tian, H. Wu, Y. Cai, J. Zhang, and P. Yuan, Opt. Lett. 36, 3173 (2011).

17. A. Marandi, B. T. Lantz, and R. L. Byer, Opt. Lett. 36, 933 (2011).

18. J. P. Dowling, Contemp. Phys. 49, 125 (2008).

19. J. Li, T. P. White, L. O'Faolain, and T. F. Krauss, Opt. Express 16, 6227 (2008).

20. S. A. Schulz, L. O'Faolain, D. M. Beggs, T. P. White, A. Melloni, and T. F. Krauss, J. Opt. 12, 104004 (2010).

21. K. M. Awan, S. A. Schulz, D. X. Liu, K. Dolgaleva, J. Upham, and R. W. Boyd, Opt. Lett. 40, 1952 (2015).

22. A. Gomez-Iglesias, D. O'Brien, L. O'Faolain, A. Miller, and T. F. Krauss, Appl. Phys. Lett. 90, 261107 (2007).

23. S. G. Johnson and J. D. Joannopoulos, Opt. Express 8, 173 (2001). 